$\underline{\text { Individual variation in memory and cognition }}$

By: Christopher N. Wahlheim and Jeffrey M. Zacks

Wahlheim, C. N., \& Zacks, J. M. (2018). Individual variation in memory and cognition. Journal of Applied Research in Memory and Cognition, 7(4), 514-517. doi:

10.1016/j.jarmac.2018.10.001

Made available courtesy of Elsevier: https://doi.org/10.1016/i.jarmac.2018.10.001

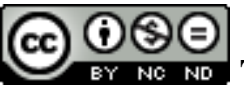
NonCommercial-NoDerivatives 4.0 International License.

\begin{abstract}
:
In this target article, Logie makes a strong case for considering variation when seeking to understand mechanisms of memory and cognition. As he puts it, "people may use their cognition in different ways to perform the same task in the laboratory and in everyday life" (Logie, 2018, p. 472). We heartily concur. Here, we underline this message by providing additional examples of inter-individual and between-group variation from research related to our areas of expertise, and extend this point by considering intra-individual variation across different instances of task performance. Our examples come from research in cognitive control, memory, everyday cognition, and cognitive aging.
\end{abstract}

Keywords: memory | cognition

\title{
Article:
}

In this target article, Logie makes a strong case for considering variation when seeking to understand mechanisms of memory and cognition. As he puts it, "people may use their cognition in different ways to perform the same task in the laboratory and in everyday life" (Logie, 2018, p. 472). We heartily concur. Here, we underline this message by providing additional examples of inter-individual and between-group variation from research related to our areas of expertise, and extend this point by considering intra-individual variation across different instances of task performance. Our examples come from research in cognitive control, memory, everyday cognition, and cognitive aging.

\section{Cognitive control}

Cognitive control is the ability to regulate thoughts and actions consistent with goal states. A core feature of cognitive control is that it varies within individuals, between individuals, and between groups of individuals. The dual mechanisms of control (DMC) framework (Braver, 2012) proposes that such variability may reflect the engagement of two qualitatively different forms of control. Proactive control reflects sustained maintenance of task goals, whereas reactive control reflects transient goal reactivation that is driven by stimulus features. Individuals differ in their habitual use of these mechanisms. In addition, individuals differ in the extent to which their 
use of these mechanisms varies across tasks and experimental conditions. Finally, groups of individuals differ; for example, it has been argued that proactive control is selectively impaired by healthy aging (Braver, 2012) and by schizophrenia (Barch \& Ceaser, 2012). In both cases, proactive control deficits are associated with altered function of the dorsolateral prefrontal cortex.

Most interesting to us, proactive and reactive control can vary not just across participants but within participants as a function of one's expectations about a task. Speer, Jacoby, and Braver (2003) trained participants to perform a working memory task in which they had to encode a list of words on each trial. In some blocks, the lists ranged in length from one to six; in other blocks, the list ranged from six to twelve. When presented with identical six-item lists, multiple behavioral markers indicated that control varied as a function of anticipated list length. In the short-list context, participants proactively rehearsed the words throughout the encoding period in anticipation of the memory probe, whereas in the long-list context they used the memory probe as a reactive cue to guide retrieval. Converging with the behavioral results, activity in the prefrontal cortex for the six-item lists varied with anticipated list length. This pattern throws into stark relief the fact that an individual may bring different algorithms to bear to solve the same problem, depending on task context.

\section{Memory}

As Logie (2018) notes, the established principles of human memory have been built largely on results aggregated across participants. He provides a thoughtful example of moving beyond such aggregation in serial recall. Another terrific case is free recall. In studies of free recall, the liberty to produce items in any order gives the researcher additional tools to assess memory mechanisms. In the history of studies of free recall, some researchers have taken the approach of modelling aggregated data whereas others have focused on individual differences, leading to substantively different conclusions. For example, context-based computational models of free recall (e.g., Healey and Kahana, 2016, Howard and Kahana, 2002, Lohnas et al., 2015, Polyn et al., 2009) attempt to explain how associations between internal context and individual items guide memory search and the organization of retrieval. These models can account very well for group-level retrieval patterns including serial position curves, which item tends to be recalled first, and the transition probabilities from item to item. However, it has been argued that free recall patterns obtained from aggregate data may lead to distorted interpretations of the mechanisms underlying performance (e.g., Hintzman, 2011), and that as a result these models may fail to account for changes in performance as individuals adopt different strategies (for an alternative approach to this issue, see Healey \& Kahana, 2014).

When researchers assess strategies on a trial-by-trial basis, they do find variation, and that performance varies with strategy (Delany \& Knowles, 2005). Strategy differences are systematically related to other individual cognitive differences, including working memory capacity (WMC), such that a model of participants with low WMC may not be an adequate description of participants with high WMC, and vice versa (Unsworth \& Engle, 2007). Finally, group differences such as age have been shown to be related to differences in strategy that are associated with differences in free recall performance (Wahlheim \& Huff, 2015). In this case, controlling for memory ability did not account for the difference in strategy nor in performance (Wahlheim, Richmond, Huff, \& Dobbins, 2016), and controlling participants' retrieval strategy 
reduced the group differences (Wahlheim, Ball, \& Richmond, 2017), suggesting that differences in retrieval strategy were causally responsible for group differences in performance.

Effects of strategy on performance are especially striking in free recall, because participants are free to vary in the order in which they retrieve items as well as in the number of items retrieved. However, even the more restricted paired associate learning task can show strong evidence for individual and group differences in patterns of performance. A classic paired associate paradigm, the A-B, A-D design, is optimized for studying effects of interference in memory. In this design, participants study two lists of cue-response pairs (often words), and are given a cued recall test for responses from one list. Importantly, one set of pairs includes the same cues (notated as A) paired with different responses in each list (notated as B for list 1, D for list 2). Often, memory for these changed pairs is worse than memory for repeated pairs, demonstrating interference (e.g., Postman \& Underwood, 1973). However, with appropriate encoding strategies, memory for changed pairs can actually be superior to memory for repeated pairs. A key mechanism that may lead to qualitative differences in performance across and within participants is reminding - incorporating the results of retrieving an earlier item when encoding a later item. If one is reminded of a previous A-B pairing when encountering an A-D pairing, this can lead to formation of a memory for the A-D experience that incorporates information about the A-B item and the relationship between the two pairs; such a representation is highly effective for subsequent memory. Across individuals, the extent to which participants report engaging in monitoring for changes and intentionally trying to remind themselves of a previous A-B pairing when seeing an A-D item explains unique variance in correct recall (e.g., Jacoby et al., 2013, Wahlheim and Jacoby, 2013). In one study, Jacoby, Wahlheim, and Kelley (2015) asked some participants to monitor during encoding for changed pairs within a single list, where others monitored for changes across the lists. The strategy of monitoring for $\mathrm{A}-\mathrm{B} / \mathrm{A}-\mathrm{D}$ change across the lists led to facilitation rather than interference. These results indicate that orienting one's encoding toward the larger experimental context leads to a qualitatively different encoding of the memoranda than does restricting one's attention to an individual list.

The processes associated with reminding have been shown to have an impact on memory beyond paired associates. Putnam and colleagues have shown that remindings have similar effects on memory for political contradictions (Putnam, Wahlheim, \& Jacoby, 2014) and memory for slide sequences in a misinformation paradigm (Putnam, Sungkhasettee, \& Roediger, 2017). Wahlheim and Zacks (2018) showed that remindings have similar effects on memory for action sequences in naturalistic movies of everyday activities.

In short, in addition to serial recall, both free recall and paired associate paradigms show clear evidence that different individuals - or even the same individuals on different occasionsmay vary in how they solve identical memory problems, leading to qualitative differences in the patterns of how they perform. These variations can be made clearer by assessing them directly and by conducting experiments in which one manipulates the hypothesized difference by instruction. These findings also extend to naturalistic stimulus materials, reinforcing Logie's (2018) point that such variations may have important impacts on performance outside the laboratory.

\section{Cognitive Aging}

When one considers how cognition changes with aging, the effects of inter-individual variation in how cognitive tasks are performed can be striking. In North America and Europe, the 
received wisdom formerly was that all people who lived long enough would eventually become demented. However, the science base now tells us this belief is wrong in multiple ways (Anderson and Craik, 2017, Park, 2000). First, dementia represents a distinct course of adult development from healthy aging. Second, trajectories of cognitive change with aging are highly variable across individuals. Third, and most important here, within-individual variability is substantial and structured.

In the domain of memory, some age differences in the effects of interference reflect differences in the occurrence of the reminding mechanism discussed in the previous section. With both word pairs (Wahlheim, 2014) and movies of everyday events (Wahlheim \& Zacks, 2018), older adults were less likely to accurately monitor changes and were less able to describe the changes later. However, when they could monitor and later describe changes, they showed benefits to memory for the events that were similar to the benefits seen in younger adults.

Another domain where individual differences in approach to a task can have a profound effect on task performance is spatial navigation. The ability to find one's way around is of course important for everyday functioning. There are large age differences in navigation performance, and although these are strongly related to age differences in nonspatial cognition (Richmond, Sargent, Flores, \& Zacks, in press), they cannot be fully explained by such general cognitive differences (Lester, Moffat, Wiener, Barnes, \& Wolbers, 2017). When learning to get around in a new environment, some people spontaneously adopt a place learning strategy, which involves learning the relations amongst locations in an environment - sometimes called a "cognitive map." Other people spontaneously adopt a response learning strategy, which involves learning a sequence of turns and landmarks associated with a route. Place learning is differentially dependent on the hippocampus and surrounding structures, which are particularly impacted by healthy aging; response learning depends differentially on the basal ganglia, which are relatively spared in healthy aging (Head \& Isom, 2010). The anatomic integrity of these structures can affect not just how well each strategy is executed, but also which is chosen (Konishi \& Bohbot, 2013). Importantly, older adults' strategy selection is sometimes maladaptive, preferring strategies that avoid place learning even for tasks where place learning is by far the more successful approach (Wiener, Condappa, Harris, \& Wolbers, 2013). For these reasons, crosssectional studies of aging and navigation that do not assess group and individual differences in strategy use may lead to a mischaracterization of the processes underlying age differences in navigation.

Whether the task is to remember words or events or to find one's way around a spatial environment, we all have "good days" and "bad days." Worse yet, it turns out that we have good times of day and bad ones. Preferred time of day turns out to be an important determiner of cognitive performance, and one that varies with age. When given a brief questionnaire asking about their preferred times to wake up, go to bed, and be active, young adults tend to identify themselves as preferring evening or being neutral, whereas the vast majority of older adults indicate a preference for the morning (May, Hasher, \& Stoltzfus, 1993). These preferences correlate with large differences in cognition: Both older and younger adults perform better at their preferred time of day on tests of explicit episodic memory (May et al., 1993), whereas effects of implicit memory show stronger effects when people are tested outside their preferred time of day (May, Hasher, \& Foong, 2005). One possibility is that these memory effects reflect circadian variation in the effectiveness of inhibitory control. Consistent with this proposal, both young and older adults show reduced performance on a number of measures of inhibitory control 
at their non-preferred time of day (May \& Hasher, 1998), though there are exceptions to this pattern (Li, Hasher, Jonas, Rahhal, \& May, 1998).

These differences have major theoretical and practical implications for theories of cognitive aging (Hasher, Zacks, \& May, 1999). The same person may approach the same task using different computational resources and dispositions, depending on whether they are at their preferred time of day. In particular, they may show greater inhibitory control at their preferred time of day. If one tests participants in the morning, older adults will be at their peak of cognitive functioning and age differences will be minimized. However, if one tests participants in the afternoon or evening, younger adults will be at their peak and larger age differences will be observed.

\section{Concluding Remarks}

We very much appreciate Logie's calling attention to the appreciation of variability in describing the mechanisms of cognition. With him, we sing the praises of studying not just group differences in central tendency but also the variability in strategies and performance profiles across individuals within a group. We hope that with this comment we have further reinforced this message, and also expanded it by pointing to the crucial role of within-individual differences in strategies and performance profiles. Let us not reduce our fellow individuals to single point estimates — as the poet Walt Whitman put it, "I am large, I contain multitudes."

\section{Conflict of Interest Statement}

The authors declare no conflict of interest.

\section{Author Contributions}

The two authors contributed equally to the conceptualization and drafting of this commentary.

\section{References}

Anderson, N. D., \& Craik, F. I. M. (2017). 50 years of cognitive aging theory. Journals of Gerontology: Series B, 72(1), 1-6.

Barch, D. M., \& Ceaser, A. (2012). Cognition in schizophrenia: Core psychological and neural mechanisms. Trends in Cognitive Sciences, 16(1), 27-34.

Braver, T. S. (2012). The variable nature of cognitive control: A dual mechanisms framework. Trends in Cognitive Sciences, 16, 106-113.

Delany, P. F., \& Knowles, M. E. (2005). Encoding strategy changes and spacing effects in the free recall of unmixed lists. Journal of Memory and Language, 52, 120-130.

Hasher, L., Zacks, R. T., \& May, C. P. (1999). Inhibitory control, circadian arousal, and age. In Attention and performance XVII: Cognitive regulation of performance: Interaction of theory and application. pp. 653-675. Cambridge, MA, US: The MIT Press.

Head, D., \& Isom, M. (2010). Age effects on wayfinding and route learning skills. Behavioural Brain Research,

Healey, M. K., \& Kahana, M. J. (2014). Is memory search governed by universal principles or idiosyncratic strategies? Journal of Experimental Psychology: General, 143, 575-596. 
Healey, M. K., \& Kahana, M. J. (2016). A four-component model of age-related memory change. Psychological Review, 123, 23-69.

Hintzman, D. L. (2011). Research strategy in the study of memory: Fads, fallacies, and the search for the "coordinates of truth". Perspectives on Psychological Science, 6, 253-271.

Howard, M. W., \& Kahana, M. J. (2002). A distributed representation of temporal context. Journal of Mathematical Psychology, 46, 269-299.

Jacoby, L. L., Wahlheim,C. N., \& Kelley,C. M.(2015). Memory consequences of looking back to notice change: Retroactive and proactive facilitation. Journal of Experimental Psychology: Learning, Memory, and Cognition, 41, 1282-1297.

Jacoby, L. L., Wahlheim, C. N., \& Yonelinas, A. P. (2013). The role of detection and recollection of change in list discrimination. Memory \& Cognition, 41, 638-649.

Konishi, K., \& Bohbot, V. D. (2013). Spatial navigational strategies correlate with gray matter in the hippocampus of healthy older adults tested in a virtual maze. Frontiers in Aging Neuroscience, 5 .

Lester, A. W., Moffat, S. D., Wiener, J. M., Barnes, C. A., \& Wolbers, T. (2017). The aging navigational system. Neuron, 95, 1019-1035.

Li, K. Z., Hasher, L., Jonas, D., Rahhal, T. A., \& May, C. P. (1998). Distractibility, circadian arousal, and aging: A boundary condition? Psychology and Aging, 13, 574.

Logie, R. H. (2018). Human cognition: Common principles and individual variation. Journal of Applied Research in Memory and Cognition, 7, 471-486.

Lohnas, L. J., Polyn, S. M., \& Kahana, M. J. (2015). Expanding the scope of memory search: Modeling intralist and interlist effects in free recall. Psychological Review, 122, 337 363.

May, C. P., \& Hasher, L. (1998). Synchrony effects in inhibitory control over thought and action. Journal of Experimental Psychology: Human Perception and Performance, 24, 363-379.

May, C. P., Hasher, L., \& Foong, N. (2005). Implicit memory, age, and time of day: Paradoxical priming effects. Psychological Science, 16, 96-100.

May, C. P., Hasher, L., \& Stoltzfus, E. R. (1993). Optimal time of day and the magnitude of age differences in memory. Psychological Science, 4, 326-330.

Park, D. C. (2000). The basic mechanisms accounting for age-related decline in cognitive function. In D. C. Park, \& N. Schwarz (Eds.), Cognitive aging: A primer (pp. 3-22). Philadelphia: Psychology Press.

Polyn, S. M., Norman, K. A.,\& Kahana, M.J.(2009). A context maintenance and retrieval model of organizational processes in free recall. Psychological Review, 116, 129-156.

Postman, L., \& Underwood, B. J. (1973). Critical issues in interference theory. Memory \& Cognition, 1, 19-40.

Putnam, A. L., Wahlheim, C. N., \& Jacoby, L. L. (2014). Memory for flip-flopping: Detection and recollection of political contradictions. Memory \& Cognition, 42, 1198-1210.

Putnam, A. L., Sungkhasettee, V. W., \& Roediger, H. L., III. (2017). When misinformation improves memory: The effects of recollecting change. Psychological Science, 28, 36-46.

Richmond, L. L., Sargent, J. Q., Flores, S., \& Zacks, J. M. (2018). Age differences in spatial memory for mediated environments. Psychology \& Aging.

Speer, N. K., Jacoby, L. L., \& Braver, T. S. (2003). Strategy-dependent changes in memory: Effects on behavior and brain activity. Cognitive, Affective, \& Behavioral Neuroscience, $3,155-167$. 
Unsworth, N., \& Engle, R. W. (2007). The nature of individual differences in working memory capacity: Active maintenance in primary memory and controlled search from secondary memory. Psychological Review, 114, 104-132.

Wahlheim, C. N. (2014). Proactive effects of memory in young and older adults: The role of change recollection. Memory \& Cognition, 42, 950-964.

Wahlheim, C. N., \& Huff, M. J. (2015). Age differences in the focus of retrieval: Evidence from dual-list free recall. Psychology and Aging, 30, 768-780.

Wahlheim, C. N., \& Jacoby, L. L. (2013). Remembering change: The critical role of recursive remindings in proactive effects of memory. Memory \& Cognition, 41, 1-15.

Wahlheim, C. N., \& Zacks,J. M. (2018). Memory guides the processing of event changes for older and younger adults. Journal of Experimental Psychology: General,. Advance online publication.

Wahlheim, C. N., Ball, H., \& Richmond, L. L. (2017). Adult age differences in production and monitoring in dual-list free recall. Psychology and Aging, 32, 338-353.

Wahlheim,C. N.,Richmond, L. L., Huff, M.J., \& Dobbins,I. G.(2016). Characterizing adult age differences in the initiation and organization of retrieval: A further investigation of retrieval dynamics in dual-list free recall. Psychology and Aging, 31, 786-797.

Wiener, J. M., de Condappa, O., Harris, M. A., \& Wolbers, T. (2013). Maladaptive bias for extrahippocampal navigation strategies in aging humans. Journal of Neuroscience, 33, 6012-6017. 